\title{
Aproximaciones a la práctica de Terapia Ocupacional en salud pública: una revisión temática
}

Approaches to Occupational Therapy practice in public health: A thematic review

\author{
Abordagens para a prática da Terapia Ocupacional em saúde pública: \\ uma revisão temática
}

\author{
Lady Dayana Ramos Ramos ${ }^{1}$ \\ Danny Giseth Herrera Páez ${ }^{2}$ \\ Paula Alejandra Sánchez López ${ }^{3}$ \\ Claudia Patricia Rojas Castillo ${ }^{4}$
}

\section{RESUMEN}

La Terapia Ocupacional ha ampliado su actuación en diferentes campos, uno de ellos, el de la salud pública. Este artículo realiza una aproximación a los antecedentes de la práctica de la profesión en ese escenario. Para identificar este recorrido, se revisaron 18 artículos, tres documentos institucionales y dos tesis de pregrado en los cuales se encontraron antecedentes teóricos, de investigación y experiencias en Sudáfrica, India, España, Canadá, Estados Unidos, Chile, Brasil, Argentina, Venezuela y Colombia. Se enfatizó en el contexto colombiano identificando aspectos relevantes en el Perfil profesional y competencias del terapeuta ocupacional. Se concluye que la Terapia Ocupacional puede generar un impacto

\footnotetext{
${ }^{1}$ Terapeuta Ocupacional. Club de Infancia. Bogotá, Colombia. Idramosr@unal.edu.co iD https://orcid.org/0000-0003-2442-1179

${ }^{2}$ Terapeuta Ocupacional. RehaSer SAS. Bogotá, Colombia. dgherrerap@unal.edu.co iD https://orcid.org/0000-0002-3103-4444

${ }^{3}$ Terapeuta Ocupacional. Clínica Universitaria Colombia. Bogotá, Colombia. paasanchezlo@unal.edu.co iD https://orcid.org/0000-0002-0275-0601

${ }^{4}$ Terapeuta Ocupacional. Magíster en Educación. Docente Universidad Nacional de Colombia. Bogotá, Colombia. cprojasc@unal.edu.co fid https://orcid.org/0000-0002-9616-4696
} 
determinante desde la atención primaria en salud, a partir de acciones a nivel individual y colectivo que contribuyen al mejoramiento de la salud de las comunidades.

\title{
PALABRAS CLAVE
}

Terapia Ocupacional, salud pública, promoción de la salud, atención primaria en salud

\begin{abstract}
Occupational Therapy has broadened its performance in different fields, including public health. This article approaches the background of Occupational Therapy practice in that field. Upon revision of 18 articles, three institutional documents and two undergraduate dissertations to identify this path, we found theoretical and research background, and experiences in South Africa, India, Spain, Canada, United States, Chile, Brazil, Argentina, Venezuela, and Colombia. The Colombian context was further explored, identifying relevant aspects of the occupational therapist's professional profile and competencies. It was concluded that occupational therapy could generate a decisive impact right from primary health care, and from both individual and collective actions, which can contribute to improving health in communities.
\end{abstract}

\section{KEY WORDS}

Occupational Therapy, public health, health promotion, primary health care

\section{RESUMO}

A Terapia Ocupacional tem ampliado sua atuação em diversos campos, um deles é o da saúde pública. Este artigo apresenta uma abordagem de antecedentes do exercício da profissão neste cenário. Para identificar este percurso, foram revisados 18 artigos, três documentos institucionais e dois trabalhos de graduação, nos quais foram encontrados antecedentes teóricos, de pesquisa e de experiência na África do Sul, Índia, Espanha, Canadá, Estados Unidos, Chile, Brasil, Argentina, Venezuela e Colômbia. Enfatizou-se, no contexto colombiano, identificações de aspectos relevantes no perfil profissional e nas competências do terapeuta ocupacional. Conclui-se que a Terapia Ocupacional pode gerar impacto decisivo a partir da atenção primária à saúde, com ações de nível individual e coletivo, que contribuam para a melhoria da saúde das comunidades.

\section{PALAVRAS-CHAVE}

Terapia Ocupacional, saúde pública, promoção da saúde, atenção primária à saúde

\section{Introducción}

El recorrido histórico de la Terapia Ocupacional comprende luchas y reivindicaciones sociales que han permitido su expansión en diferentes niveles, así como construir saberes sobre las ocupa- ciones y lo que estas implican a nivel individual y colectivo. Este abordaje propio de la profesión, a través de la lectura de las personas, sus ocupaciones y la relación con su contexto o ambiente, abre 
una serie de posibilidades de acción en diversos escenarios, como la participación en la estrategia de atención primaria en salud desde una perspectiva colectiva en salud pública.

Por medio de la Declaración de Alma-Ata, generada en el marco de la Conferencia Internacional sobre Atención Primaria en Salud, en 1978, la Organización Mundial de la Salud -OMS recomendó a todos los sectores y niveles de gestión desarrollar y mantener la atención primaria en salud como estrategia para promover y proteger la salud de todas las personas, definiéndola como:

La asistencia sanitaria esencial basada en métodos y tecnologías prácticos, científicamente fundados y socialmente aceptables, puestos al alcance de todos los individuos y familias de la comunidad, mediante su plena participación y a un costo que la comunidad y el país pueden soportar en todas y cada una de sus etapas de desarrollo con un espíritu de autodependencia y autodeterminación. (1978, p.1)

Desde entonces, la atención primaria en salud se convirtió en el marco social y político que adoptaron muchos gobiernos como expresión de voluntades para lograr las metas en salud. Asimismo, se han encontrado otras perspectivas para comprenderla. Por ejemplo, la versión que reclaman corrientes como la medicina social proponen una visión transformadora y profunda, en una lógica integral que reconozca las desigualdades en salud y se enfoque en la transformación de sus causas estructurales (Molina, 2012, p.19).
Luego de 35 años desde la Conferencia de Alma-Ata, la Organización Mundial de la Salud ha aceptado que el cumplimiento de esos mínimos está lejos de ser una realidad en muchas partes del mundo y que, por el contrario, la distancia entre países ricos y pobres han crecido de manera considerable (Jaramillo, 2012, p.11). Por esta razón, las desigualdades en salud se han ubicado como un punto central dentro del balance sobre la implementación de la atención primaria en salud, ya que, como lo afirma Jaramillo, la estrategia fue mal interpretada, pues se confundió "con atención de primer nivel y escasa capacidad resolutiva" (2012, p.12).

Es por ello que resulta importante retomar la corriente que comprende la atención primaria en salud como un conjunto de procesos que se deben orientar a la transformación de las causas estructurales de la injusticia y la desigualdad, donde las respuestas sociales al proceso salud-enfermedad se ubican en un campo de conflictos que reflejan relaciones de poder conducentes a modos de vida con enfermedades evitables y, en últimas, a inequidades en la atención a la salud y la enfermedad (Molina, 2012).

En ese sentido, la perspectiva colectiva de la salud se relaciona y complementa con una nueva meta, que incluye dar un paso importante para superar las desigualdades en salud de manera más articulada con la construcción colectiva de condiciones de vida digna, pues es clave trabajar alrededor de la relación entre salud, equidad y desarrollo (Jaramillo, 2012, p.12).

Por lo tanto, es relevante entender que la trayectoria de la salud pública y 
su relación con la Terapia Ocupacional en diferentes países también refleja pugnas de diferente naturaleza y es el resultado de sus contextos sociales, políticos y culturales, y que el enfoque de salud y las posibilidades de desarrollo de la profesión son causas estructurales que determinan la salud. En consecuencia, documentar, rastrear y explorar diversas experiencias de terapeutas ocupacionales en salud pública permite identificar elementos de articulación en el hacer, poner en diálogo formas y reflexiones que contribuyan a enriquecer la práctica en este campo en diferentes contextos, con miradas territoriales y comunitarias desde la atención primaria en salud que, a su vez, permitan pequeñas transformaciones estructurales.

De esta manera, esta revisión estuvo orientada a brindar una aproximación a los antecedentes y trayectorias de la práctica de la Terapia Ocupacional en salud pública en varios países del mundo, así como a identificar elementos para la comprensión del hacer de la profesión en dicho campo en Colombia.

\section{Metodología}

Se realizó una búsqueda de literatura empezando con los descriptores en ciencias de la salud - DeCS Terapia Ocupacional y salud pública. Sin embargo, dado que los resultados fueron escasos, se decidió ampliar la búsqueda con conceptos relacionados, incluyendo los descriptores atención primaria de salud, promoción de la salud y prevención de enfermedades.

Con estos descriptores, se buscaron artículos publicados entre los años 2000 y 2019 en revistas de Terapia Ocupacional y otras que cubren el campo de la profesión, estas fueron: Revista de Terapia Ocupacional Da Universidade de São Paulo, Revista Argentina de Terapia Ocupacional, Revista Chilena de Terapia Ocupacional, Revista Ocupación Humana, Revista Electrónica de Terapia Ocupacional Galicia (TOG), American Journal of Occupational Therapy, Revista Colombiana de Rehabilitación y Revista de la Facultad de Medicina, y en las bases de datos OTseeker, Scielo, Electronic Library Online, Pubmed y Biblioteca Virtual en Salud (BVS - Portal de Revistas Científicas en Ciencias de la Salud).

Se revisaron publicaciones en español, inglés y portugués. Solo se tuvieron en cuenta aquellas propias de Terapia Ocupacional, cuyo título o resumen incluyera los descriptores nombrados anteriormente.

También se revisaron las páginas web oficiales de algunas organizaciones gremiales de terapeutas ocupacionales: Council of Occupational Therapists for the European Countries, Occupational Therapy Association of South Africa y Colegio Colombiano de Terapia Ocupacional, buscando documentos institucionales relacionados con el tema; aunque se indagó en otras páginas web de asociaciones de Terapia Ocupacional, esta exploración no fue exhaustiva y se decidió tomar las mencionadas anteriormente.

Por último, se revisaron dos trabajos de grado referidos en encuentros académicos, uno de ellos, a través de sus autores.

Una vez escogidos los textos, se tuvieron en cuenta los siguientes elementos para el análisis: procedencia geo- 
gráfica, rol del terapeuta ocupacional, enfoque u objetivos de atención (si se mencionan), acciones desarrolladas y resultados esperados.

\section{Resultados}

En total se identificaron y analizaron 18 artículos, tres documentos institucionales y dos trabajos de pregrado con antecedentes de Sudáfrica, India, España, Canadá, Estados Unidos, Chile, Argentina, Brasil, Venezuela y Colombia, publicados entre los años 2003 y 2018. No se encontraron documentos entre el 2000 y 2002 que cumplieran con los criterios de selección (tabla 1).

A continuación se presentan los resultados de acuerdo con su procedencia geográfica.

\section{Europa, Asia, África y Norteamérica}

En Europa, desde el Council of Occupational Therapists for the European Countries -COTEC, conformado por las agremiaciones de 33 países, preocupados por las dificultades en la participación y el reconocimiento de los aportes de terapeutas ocupacionales en los equipos de atención primaria, se toma la decisión de generar redes para fortalecer esos procesos. De esta manera, construyen el documento Occupational Therapy perspective on integrated care (COTEC, 2016), donde se resalta la participación de terapeutas ocupacionales que se centran en la "habilitación en la vida diaria" y en la participación de personas de todas las edades, sus cuidadores y diferentes grupos en la sociedad. En este documento se relacionan programas de Terapia Ocupacional dirigidos a la población con Alzhéimer, Parkinson y personas que han sufrido accidentes cerebro-vasculares, pues se ha demostrado que tales intervenciones aumentan la calidad de vida y mejoran el rendimiento autopercibido en las actividades de la vida diaria.

Así mismo en India, debido a la sobrepoblación en Vellore, no todas las personas pueden acceder a la atención en salud. Por ello, con la colaboración del Asia Pacific Occupational Therapists Regional Group (APOTRG) se creó el programa Community Health and Development -CHAD, que consiste en equipos móviles de salud que hacen visitas semanales a las familias y personas que les son asignadas en cada comunidad (Kidd, 2018). En estos equipos participan terapeutas ocupacionales que desarrollan programas para facilitar el acceso al empleo; para adultos mayores, quienes presentan altas tasas de suicidio; programas de fortalecimiento de habilidades motoras finas para niños y niñas, y otros enfocados en la prevención de enfermedades.

Por otro lado, en Sudáfrica, la Occupational Therapy Association of South Africa -OTASA (2018), desde su visión sobre la práctica de Terapia Ocupacional en la atención primaria en salud, propone dar un nuevo enfoque al sistema de salud de ese país, articulando el compromiso de la profesión con la equidad y la justicia social a través de una transformación de la atención asistencialista a una más integral. La propuesta parte de la reorientación de los programas de formación de pregrado, de manera que las y los profesionales de la salud puedan responder al enfoque de atención primaria. 
Tabla 1. Procedencia y periodos abarcados en documentos revisados.

\begin{tabular}{|l|c|}
\hline \multicolumn{1}{|c|}{ Ubicación geográfica } & Periodo \\
\hline $\begin{array}{l}\text { Europa, Asia, África y Norteamérica: } \\
\text { Estados Unidos, Canadá, España, India y } \\
\text { Sudáfrica. }\end{array}$ & 2009 a 2016 \\
\hline $\begin{array}{l}\text { Latinoamérica: } \\
\text { Argentina, Chile, Brasil y Venezuela. }\end{array}$ & 2004 a 2018 \\
\hline Colombia & 2003 a 2018 \\
\hline
\end{tabular}

Fuente: elaboración propia.

De acuerdo con Naidoo et al. (2016), el sistema de salud sudafricano reconoce que la atención en salud es un derecho de todas las personas y busca garantizar el acceso a este para toda su población, por ello, se han creado equipos de asistencia a nivel municipal, de especialistas locales y de salud escolar. Estos equipos, conformados por personal de Enfermería, Medicina, Psicología, Trabajo Social, Nutrición y Terapia Ocupacional, realizan labores de promoción de una vida saludable; prevención, detección temprana, tratamiento y manejo de enfermedades, y rehabilitación basada en la comunidad.

Entre tanto en España, la experiencia de las y los terapeutas ocupacionales en la atención primaria en salud se ha centrado en establecer equipos que tienen por objetivo mejorar el acceso a servicios de calidad, por medio de la atención integral. Lombardo y Ro- mero (2012) destacan que, además de los aportes a las personas que presentan problemas de salud, es fundamental la participación de la comunidad en la planificación de proyectos y programas de promoción de la salud y prevención de la enfermedad. A manera de ejemplos, citan los programas de preparación para la jubilación, de orientación sobre indicaciones y contraindicaciones para la realización de actividades básicas e instrumentales de la vida diaria, y las visitas domiciliarias que facilitan el desempeño ocupacional.

En cuanto a Norteamérica, según Hammel et al. (2015), en Estados Unidos, gracias a la Ley de Protección al Paciente y Cuidado de Salud Asequible ${ }^{5}$, como estrategia de atención primaria integral existen grupos interdisciplinarios en los que participan terapeutas ocupacionales y que desarrollan programas orientados a mejorar el acceso a la

${ }^{5}$ Patient Protection and Affordable Care Act (2010), conocida como Obamacare. 
atención y disminuir gastos al sistema de salud, por medio de estrategias que buscan evitar la readmisión a los hospitales, manejar síntomas en casa para personas con enfermedades crónicas y apoyar a las familias.

Por otro lado en Canadá, la Society of Alberta Occupational Therapists - SAOT (2016), en el documento The role of Occupational Therapy in primary care, reconoce que la salud es el resultado de lo que las personas hacen o no en la cotidianidad y que la Terapia Ocupacional puede desempeñar un papel importante en la promoción de la salud y el bienestar a través de la participación en actividades cotidianas y significativas. Siguiendo a Wilcock, esta Asociación propone cuatro niveles de intervención para mejorar o mantener la salud y el bienestar en la atención primaria en salud por parte de terapeutas ocupacionales. El primero es la prevención de conductas que puedan producir enfermedades; el segundo, la prevención de una mayor progresión de la enfermedad; el tercero, el mantenimiento de la salud y el bienestar en personas que viven con enfermedades crónicas, y el último, la preservación de la calidad de vida de personas con enfermedades terminales (SAOT, 2016).

\section{Latinoamérica}

Desde Argentina, Pellegrini (2004) refiere que a la hora de hablar de promoción de la salud y trabajo comunitario, la Terapia Ocupacional participa en acciones comunitarias identificando aquellas condiciones en el ambiente físico, cultural y social que hacen la vida más difícil a cierto grupo de personas, y busca soluciones apropiadas que tengan en cuenta el panorama políti- co que permea el sistema de salud de cada país. Afirma que las y los terapeutas ocupacionales deben formular una propuesta que focalice las necesidades de salud a largo plazo y, a su vez, desarrolle comportamientos saludables para mejorar la salud y minimizar los costos asociados a condiciones de enfermedad y discapacidad.

En Chile, Chaparro et al. (2005) mencionan que la formación de terapeutas ocupacionales en relación con la promoción y la prevención ha permitido desarrollar prácticas comunitarias en el nivel básico de atención desde una visión integral del ser humano como agente de salud, teniendo en cuenta los estilos de vida y el medio ambiente. De la misma forma, Palacios y Pino (2016) proponen una relación entre Terapia Ocupacional y salud pública que parta de la lectura de los determinantes sociales y culturales a través de la intervención comunitaria y la realización de diagnósticos participativos y epidemiológicos en los programas locales de promoción. En ese sentido, destacan la participación de Terapia Ocupacional en programas ligados a la infancia, la adultez, la salud mental, la promoción de salud, la rehabilitación basada en la comunidad, la gestión y la administración. Adicionalmente, Aróstica et al. (2018) resaltan la necesidad de hacer una lectura particular de cada territorio, de manera que se reconozcan factores culturales que pueden afectar las acciones a realizar.

Desde Brasil, Barata et al. (2015) proponen implementar acciones de promoción de la salud y clínicas, así como articular y mapear redes y estrategias colectivas realizadas en escuelas, domicilios y otros espacios de la comunidad que sirven como apoyo para 
la promoción de la salud. De la misma forma, Silva y Oliver (2017) coinciden en la necesidad de que, desde Terapia Ocupacional, se desarrolle un trabajo colectivo, integrado, interprofesional e intersectorial que apoye la cualificación de las acciones, así como el acceso y la integralidad de los cuidados en la atención primaria en salud.

Entre tanto, Munguba et al. (2018) hacen un Ilamado a ir más allá de las intervenciones tradicionales en salud e impulsar la innovación y la investigación para hacer contribuciones teóricas y metodológicas al trabajo de la Terapia Ocupacional en otros sectores, y así lograr una mayor participación social en la sociedad capitalista y globalizada actual. En últimas, las y los terapeutas ocupacionales deben abordar la cuestión social, esto significa identificar desigualdades y desarrollar intervenciones que no se limiten a la mejora individual, sino a las condiciones colectivas de salud.

Por su parte Bianchi (2018), también desde Brasil, reflexiona sobre la importancia del territorio y las acciones colectivas en el ejercicio de la Terapia Ocupacional. Concluye que visualizar el territorio no es solo una opción de intervención, es también un instrumento que potencializa la atención terapéutica, la inserción y la participación social. Estos planteamientos toman fuerza cuando las acciones de Terapia Ocupacional se articulan con la vida cotidiana de los sujetos y las comunidades.

Narh (2014), en Venezuela, rescata la concepción humanista que considera ha hecho parte de la formación de terapeutas ocupacionales desde los inicios de la profesión, de manera que la prevención de la enfermedad y la promo- ción de la salud que realizan deban ir más allá de las prácticas institucionalizadas y ser ejercidas en los escenarios comunitarios, logrando entender la cotidianidad de las personas y la manera como las comunidades construyen bienes comunes y justicia social. De esta forma, el papel de la Terapia Ocupacional en Latinoamérica, según la autora, no es solamente asistencial, es también social, ámbito en el que puede hacer varias contribuciones desde la promoción de la salud, aumentando el acceso a los servicios de salud, desarrollando ambientes saludables, fortaleciendo los grupos y recursos comunitarios, promocionando comportamientos saludables e incrementando los conocimientos acerca de la salud.

\section{Colombia}

Según Peñas (2003), la Terapia Ocupacional se ha venido abriendo campo en el trabajo en comunidad, donde se propone una relación con la salud pública que exige desarrollar habilidades y destrezas de intervención a nivel comunitario en los niveles de promoción del bienestar ocupacional, prevención y rehabilitación de alteraciones o disfunciones ocupacionales. Esta autora propone algunos conceptos que considera tienen relación con la labor de la profesión en salud pública: salud colectiva; interdisciplinariedad; política pública; atención primaria en salud desde la organización y la participación de la comunidad, y rehabilitación basada en comunidad. Lo comunitario es planteado como eje transversal.

Desde otra mirada, Arango et al. (2013) realizan un análisis del papel de las y los terapeutas ocupacionales en el marco de las reformas a los sistemas de 
salud, centrándose en el caso de Colombia. Mencionan que, como profesionales de salud, es importante adoptar un enfoque para entender a la persona no solo desde el momento en el que llega al acompañamiento por Terapia Ocupacional, sino como resultado de un proceso histórico que ha influenciado sus condiciones de vida, su acceso al sistema de salud y la estructura de este.

De esta manera, proponen al gremio de Terapia Ocupacional preguntarse sobre la relación salud-ocupación y la ocupación determinada por elementos del contexto físico, ambiental y social, ya que "entre los elementos que determinan la salud es perentorio destacar la preponderancia de la ocupación que los sujetos desempeñan" (Arango et al., 2013, p.7), y teniendo en cuenta que esta influye en la posición del individuo en la estructura social y en su exposición a riesgos laborales o cotidianos, facilita o restringe el acceso a recursos sanitarios, conlleva a diferentes niveles de estrés y favorece o dificulta la adopción de hábitos saludables.

En ese sentido, estos autores señalan que "el terapeuta ocupacional sigue siendo considerado como un rehabilitador que actuaría cuando se configure una disfunción que afecte el desempeño ocupacional" (Arango et al., 2013, p.12), pues, aseguran, a pesar de los avances en ámbitos diferentes a la clínica, se mantiene el modelo biomédico tras el afán de reconocimiento de la profesión. No obstante, manifiestan que:

Con el conocimiento que se posee sobre la ocupación humana y su valor, el desempeño ocupacional y su dinámica, es factible aparecer como actores fuertes antes de la disfunción, ser agentes del primer nivel con actividades de promoción de la salud y prevención de la enfermedad, dirigir la mirada a los determinantes de la salud y la calidad de vida de individuos y poblaciones. (2013, p.12)

En ese mismo año, Garcés y Sabogal (2013), desde su trabajo de grado de pregrado en la Universidad del Valle, aportan a la determinación de las acciones de Terapia Ocupacional en la atención primaria en salud. En esta investigación presentan un panorama general de la normativa vigente, la historia de la atención primaria en salud y la opinión de profesionales en Terapia Ocupacional con experiencia en el campo. De esta manera, destacan que:

Las acciones de la Terapia Ocupa-
cional en la estrategia de atención
primaria en salud deben guiarse bajo
el enfoque amplio de la misma (Con-
ferencia Alma-Ata), puesto que el ob-
jeto de la Terapia Ocupacional es fa-
vorecer el desempeño ocupacional de
las personas y colectividades, en este
caso, y bajo esta estrategia sus accio-
nes están guiadas hacia la promoción
de la participación en ocupaciones
significativas que generen un estado
de bienestar, previniendo así situacio-
nes como el aislamiento y poca parti-
cipación en las actividades cotidianas.
(Garcés y Sabogal, 2013, p.66)

A su vez, respecto a lo que podría guiar las acciones profesionales en el campo, señalan como principio:

Capacidad (...) para entender y comprender las interacciones que se dan desde el nivel individual, familiar, social y cultural entre las personas y colectividades con el fin de aportar 
elementos conceptuales, filosóficos y epistemológicos a la comprensión del desempeño y la ocupación en el actual contexto social, político, económico, cultural y de conflicto armado y social que vive el país. (Garcés y Sabogal, 2013, p.65)

Por su parte, el Colegio Colombiano de Terapia Ocupacional y el Ministerio de Salud y Protección Social generaron el documento Perfil profesional y competencias del terapeuta ocupacional (Navas y Tenorio, 2016) que incluye dominios, competencias y criterios de desempeño para terapeutas ocupacionales en Colombia; dentro de los dominios se encuentran la promoción de la salud y la salud pública. A continuación se citan, de forma textual, las competencias específicas para estos dominios.

Promoción de la salud

-Gestionar prácticas que impacten las determinaciones sociales, políticas y económicas de la salud de personas y colectivos $y$, que fomenten o mejoren la participación en ocupaciones saludables y satisfactorias.

-Aportar a la transformación positiva de las determinaciones sociales, políticas y económicas de la salud de la población colombiana, vinculadas a la relación bienestar- ocupación. (p.20)

Salud Pública

-Aportar en el análisis de situación poblacional a través de la comprensión de la ocupación en cualquiera de sus dimensiones y la relación con la salud y la vida en los territorios a los que se refiera.
-Aportar en la formulación de políticas públicas que promuevan la participación de las personas y las comunidades en ocupaciones saludables y significativas. (p.28)

Finalmente, Arango et al. (2018) plantean una serie de retos para la Terapia Ocupacional en salud pública y retoman la idea de profundizar en "la ocupación humana como determinante esencial de la promoción y la prevención de la enfermedad" (p.382). Señalan como primer reto entender la salud no solo desde la enfermedad y empezar a hablar de ella en positivo, como bienestar de individuos y colectivos. Afirman que las y los terapeutas ocupacionales pueden cumplir su labor siendo promotores de salud, partiendo de que la ocupación proporciona mecanismos para suplir necesidades de supervivencia $y$ salud, y la actividad con sentido y significado puede prevenir la enfermedad; en definitiva, entendiendo la ocupación como eje de la calidad de vida.

\section{Discusión}

Los hallazgos evidencian que la trayectoria de Terapia Ocupacional en salud pública es el resultado de la interacción entre factores sociales, políticos y culturales de cada país, pues son estas condiciones las que posibilitan el desarrollo de la profesión y enmarcan su hacer en determinados enfoques y niveles de atención, de acuerdo con los lineamientos gubernamentales que se adopten en cada territorio.

De esta manera, dar cuenta de las limitaciones de esta revisión pasa por entender las diferencias entre los contex- 
tos $y$, por ende, los conceptos utilizados para enmarcar la práctica en un campo de acción. Por ejemplo, la búsqueda de literatura arrojó pocos resultados al usar solo el descriptor salud pública, siendo necesario acudir a otros que encuadran el hacer en la atención primaria en salud, con diferencias en los niveles de atención. Por otra parte, en países como Brasil y Argentina los resultados podrían ampliarse usando los conceptos salud colectiva o comunidad. También es importante mencionar que la revisión se limita a documentos disponibles en internet y en las fuentes seleccionadas, de manera que podrían encontrarse experiencias y aportes importantes fuera de esas revistas científicas y portales de búsqueda, tanto en otros países como en Colombia.

Se rescata la presencia de terapeutas ocupacionales en equipos interdisciplinarios en varios países, creados por iniciativa de los Estados y que buscan responder a las necesidades de las comunidades desde un enfoque de atención primaria en salud. Sin embargo, las diferencias en prácticas, conceptos y estrategias se hacen evidentes entre regiones y países. En Latinoamérica se empieza a hablar del trabajo en áreas rurales y con la participación de las comunidades, de conocimiento de derechos y empoderamiento, partiendo del propósito de transitar de un modelo biomédico asistencialista a un enfoque biopsicosocial de bienestar. Entre tanto, en Norteamérica se mantiene una tendencia hacia la asistencia que responde a situaciones de enfermedad, pero no a procesos de transformación de las causas estructurales de las desigualdades en salud.

En regiones más distantes se observan matices en las prácticas y en los li- neamientos y posicionamientos de las asociaciones. Así, mientras en Sudáfrica e India se mencionan experiencias comunitarias con equipos profesionales que llegan a los territorios desde el primer nivel de atención, en Europa se encuentra un poco de los dos enfoques; por un lado, programas orientados a aumentar la calidad de vida en personas con una patología de base, y por otro, programas de prevención y planificación de proyectos con participación de la comunidad.

En Colombia se encuentran aportes teóricos, de comprensión y de identificación de elementos en el hacer que ubican al terapeuta ocupacional como agente promotor de la salud y en la prevención de la enfermedad, entendiendo a cada persona como resultado de su desarrollo histórico y sujeto dentro de sus contextos cotidianos y comunitarios. Se asocia la atención primaria en salud a la organización y la participación de la comunidad, dirigida a los determinantes sociales de la salud a nivel individual y colectivo.

Así mismo, es importante mencionar que las acciones en este campo se encuentran en construcción y transformación, pues las posibilidades en este ámbito, con lo comunitario como eje transversal, también son resultado de las dinámicas sociales, políticas y culturales que atraviesa el país, y estas han traído consigo lineamientos que requieren aún llegar a las causas estructurales de las desigualdades en salud.

Es necesario reconocer, sin embargo, que dadas las limitaciones de esta revisión, en las fuentes seleccionadas no se encontraron experiencias desarrolladas en el ámbito de la salud pública colombiana con participación de terapeutas 
ocupacionales. Ello indica la necesidad de posteriores investigaciones.

Aun así, es importante apropiar conocimientos, prácticas y experiencias en pro de ampliar y fortalecer los diferentes campos de acción de la Terapia Ocupacional. En esa medida, resulta imperativo que las y los terapeutas ocupacionales continuemos asumiendo papeles adicionales a la intervención asistencial, rompiendo esquemas y yendo más allá de lineamientos tradicionales u oficiales. Es importante motivar y desarrollar desde otras experiencias en "el lugar del territorio, con los conocimientos que ayudan en las transformaciones de las vidas, con las propuestas innovadoras, creativas, justas, para que efectivamente las vidas y los lugares donde interactuemos encuentren el sello de la participación de un terapeuta ocupacional" (García, 2016, p.89).

En este sentido, también es necesario fortalecer los programas de formación en Terapia Ocupacional, entendiendo su papel fundamental como guía de la práctica profesional, pues, siguiendo a Arango et al., hay que pensar "si bastará con incluir en el currículum de los programas un módulo o un curso de salud pública" (2018, p.381) y si se requiere abrir o ampliar escenarios experienciales en los que la práctica no se enmarque en la asistencia, si no en cómo se asume el ejercicio profesional desde un territorio particular y en una perspectiva de salud pública. De esta manera, la academia podría aportar eficazmente a la construcción de la profesión, teniendo en cuenta, además, su lugar en la investigación y en la sistematización de experiencias y reflexiones sobre el quehacer de la Terapia Ocupacional.
Lo anterior es una invitación a tomar postura y a actuar en las transformaciones y en los escenarios políticos, sociales y culturales que atraviesan nuestro país desde la academia y los lugares de incidencia en el ejercicio profesional.

\section{Conclusiones}

Desde países distantes y cercanos, esta revisión permitió identificar el papel de la Terapia Ocupacional en diferentes escenarios en los que se asume la promoción de la salud y la prevención de la enfermedad como pilares a la hora de hablar de salud pública. A su vez, dio pie a reconocer que estos escenarios pueden ser promovidos por los Estados a través de políticas públicas y programas de instancias gubernamentales.

La trayectoria de la profesión en salud pública en Colombia es también el resultado de los lineamientos adoptados en cada gobierno y de la formulación de políticas públicas regresivas en cuanto al derecho a la salud. Por ello, hoy en día se requiere que las y los terapeutas ocupacionales asuman liderazgos, actúen y ocupen el lugar; ocupar un lugar implica ocupar todos los lugares donde construyamos con las comunidades conocimientos sobre la ocupación humana en función del bienestar de las personas. En este sentido, poner los conocimientos en función implica participar en la formulación de políticas públicas, en proyectos comunitarios, desarrollar acciones que permitan el trabajo colaborativo con las personas y las comunidades en sus territorios y cotidianidades. Por ejemplo, el contexto del posacuerdo "podemos verlo como una nueva puerta de oportunidad para fortalecer nuestro actuar profesional, pensado y vinculado 
con los problemas sociales, económicos y culturales que afectan a grandes grupos humanos históricamente excluidos en nuestro país" (Rojas, 2017, p.3).

Finalmente, es imperativo construir reflexiones, posibilidades y acciones desde una mirada interseccional que permita articular propuestas sociales y experiencias de acción colectiva que contribuyan a la transformación de las desigualdades en salud y aporten en el camino de lucha por el derecho a la salud y a la salud pública. Lo anterior, desde la atención primaria en salud, pero también a través de acciones de rehabilitación a nivel individual y colectivo que tengan como base la diversidad social y la participación de los sectores sociales excluidos, de manera que aporten a la salud de las comunidades para la construcción de una sociedad justa y equitativa.

\section{Referencias}

Arango, J., Correa, Y., Luna, J., Campos, A. y Molano, A. (2013). El terapeuta ocupacional en el marco de las reformas de los sistemas de salud: reflexiones desde el caso Colombia. Revista Ocupación Humana, 13 (2), 7-23. https://doi. org/10.25214/25907816.55

Arango, J., Correa, Y. y Méndez, J. (2018) . Retos de la terapia ocupacional en salud pública . Revista Facultad de Medicina, 66 (3), 375-83. https://doi.org/10.15446/ revfacmed.v66n3.63438

Aróstica, N., Garrido, B., Saavedra, J. y Salinas, M. (2018). Experiencias de terapeutas ocupacionales con indígenas: una mirada desde los equipos rurales de rehabilitación [Seminario de título, Universidad de la Frontera]. Biblioteca Digital de la Universidad de la Frontera. http://bibliotecadigital.ufro.cl/?a=view\&item $=1505$

Barata, M., Nóbrega, K., Jesus, K., Lima, M. L. y Facundes, V. L. (2015). Rede de cuidado a crianças e adolescentes em sofrimento psíquico: ações de promoção à saúde. Revista de Terapia Ocupacional da Universidade de São Paulo, 26(2), 225-233. https://doi.org/10.11606/issn.2238-6149. v26i2p225-233

Bianchi, P.C. (2018). Dos entrecruzamentos da Terapia Ocupacional e o território: reflexões a partir da prática profissional na Atenção Básica em Saúde. Revista Argentina de Terapia Ocupacional, 4(1), 40-46. http://www.revista.terapia-ocupacional.org.ar/RATO/ 2018jul-rato.pdf

Chaparro, R., Fuentes, J., Moran, D. y Mujica, N. (2005). La terapia ocupacional en la intervención en salud comunitaria: el modelo educativo. Revista Chilena de Terapia Ocupacional, (5), 69-79. https://auroradechile.uchile.cl/index.php/RTO/article/view/168

Council of Occupational Therapists for the European Countries [COTEC] (2016). Occupational Therapy perspective on integrated care. http://www.coteceurope.eu/wp-content/uploads/2015/07/ Occupational-Therapy-perspective-on-Integrated-Care.pdf

Garcés, A. y Sabogal, J. (2013). Aproximación a las acciones de la terapia ocupacional en la atención primaria en salud en Colombia [trabajo de grado de pregrado no publicado]. Escuela de Rehabilitación Humana, Universidad del Valle. 
García, S. (2016). Terapeuta ocupacional: sujeto político. Revista Ocupación Humana, 16 (1), 84-90. https://doi. org/10.25214/25907816.16

Hammel, J., Magasi, S., Mirza, M., Fischer, H., Preissner, K., Peterson, E. y Suárez, Y (2015). A scholarship of practice revisited: Creating community-engaged occupational therapy practitioners, educators, and scholars. Occupational Therapy In Health Care, 29(4), 352-369. https://doi. org/10.3109/07380577.2015.1051690

Jaramillo, G. (2012). Presentación. En: P. Ramírez (ed.), Atención primaria en salud: un camino hacia la equidad (p.p 11-13). Corporación para la Salud Popular Grupo Guillermo Fergusson y Secretaría Distrital de Salud de Bogotá.

Kidd, E. (2018). Occupational Therapy in India. http://www.kumc.edu/Documents/ international/Ellie\%20Kidd \%20India \%20 Presentation\%20.pdf

Lombardo, A. y Romero, A. (2012). Terapia ocupacional en la cartera de servicios de atención primaria: ¿es posible?. Revista Electrónica de Terapia Ocupacional Galicia, TOG, 9(16), 8-30. http://dialnet.unirioja.es/servlet/articulo? codigo $=4221031$

Molina, N. (2012) Antecedentes y desarrollo de la APS. En: P. Ramírez (ed.), Atención primaria en salud: un camino hacia la equidad (p.p 18-37). Corporación para la Salud Popular Grupo Guillermo Fergusson y Secretaría Distrital de Salud de Bogotá.

Munguba, M., Malfitano, A. y Lopes, R. (2018). The debate over the social question in occupational therapy: An integrative review. Cadernos Brasileiros de Terapia Ocupacional, 26(4), 892-903. https://doi. org/10.4322/2526-8910.ctoar1748
Naidoo, D., Van, J. y Joubert, R. (2016). Exploring the occupational therapist's role in primary health care: Listening to voices of stakeholders. African Journal of Primary Health Care \& Family Medicine, 8(1), 1-9. https://doi.org/10.4102/phcfm. v8i1.1139

Narh, E. (2014). Promoción y prevención en terapia ocupacional. Cuadernos de la Escuela de Salud Pública. 2(88), 59-65. http://saber.ucv.ve/ojs/index.php/rev edsp/article/view/8216/8122

Navas, A. y Tenorio, L. (coord.). (2016). Perfil profesional y competencias del terapeuta ocupacional. Colegio Colombiano de Terapia Ocupacional y Ministerio de salud y Protección Social. https://doi. org/10.25214/ccto. 1

Occupational Therapy Association of South Africa [OTASA] (2018). Occupational Therapy Association of South Africa position statement on rehabilitation. The South African Journal of Occupational Therapy, 47(3), 63-64. https:// doi.org/10.17159/2310-3833/2017/ v47n3a10

Organización Mundial de la Salud [OMS] (1978). Atención Primaria de Salud. Informe de la Conferencia Internacional sobre Atención Primaria de Salud, Alma Ata, URSS. http://www.paho.org/spanish/ dd/pin/alma-ata declaracion.htm

Palacios, M. y Pino, J. (2016). Reconstrucción del sentido de comunidad y ocupaciones colectivas: experiencias de transformación de prácticas de salud primaria rural. En: S.Simó, A. Guajardo, F. Oliver, S. Galheigo y S. García-Ruiz. Terapias Ocupacionales desde el Sur (pp. 415-432). Universidad de Santiago de Chile 
Pellegrini, M. (2004, diciembre). Terapia Ocupacional en el trabajo de salud comunitaria. Terapia-Ocupaiconal.com. http:// www.terapia-ocupacional.com/articulos/ Salud-Comunitaria.shtml

Peñas, O. (2003). La intervención comunitaria en terapia ocupacional: un acercamiento desde la salud pública. Revista Colombiana de Rehabilitación, 1(2), 21-27. https://doi.org/10.30788/RevColReh.v2.n1.2003.242

Rojas, C. (2017). Terapia Ocupacional en el posacuerdo. Retos para una profesión que se transforma. Revista Ocupación Humana, 17(1), 3-6. https://doi. org/10.25214/25907816.150

Silva, R., y Oliver, F. (2017). Trajetória docente e a formação de terapeutas ocupacionais para atenção primária à saúde. Interface: Communication, Health, Education, 21(62), 661-673. https://doi. org/10.1590/1807-57622016.0024

Society of Alberta Occupational Therapists [SAOT] (2016). The role of occupational therapy in primary care. https://www. saot.ca/wp-content/uploads/2016/10/ The-Role-of-OT-in-Primary-Health-Care_November-2016.pdf 